\title{
Industry Supported Improved Design of DEMO BoP for HCPB BB Concept with Energy Storage System
}

Evaldas Bubelis, Wolfgang Hering, Sara Perez-Martin Institute for Neutron Physics and Reactor Technology, Karlsruhe Institute of Technology (KIT), EggensteinLeopoldshafen, Germany

The work described in this paper was performed in the frame of the European Fusion Programme (EUROfusion), Power Plant Physics and Technology (PPPT) section, Balance of Plant (BoP) work package for EU DEMO Fusion Power Plant (FPP).

DEMO BoP mainly consists of the Primary Heat Transfer System (PHTS), the Intermediate Heat Transfer System (IHTS) that uses HITEC salt as coolant and is equipped with thermal Energy Storage System (ESS) to cope with DEMO pulsed operation, and the Power Conversion System (PCS). The proposed DEMO BoP configuration is capable of producing electricity continuously through the pulse time ( 2 hours), as well as dwell time (10 $\mathrm{min}$ ) of the plant operation. The efficiency of the proposed Rankine cycle of the PCS is obtained to be not less than $39 \%$. While elaborating the improved DEMO BoP design, KIT developed a plant model using the industrial code EBSILON®. The development process of the DEMO BoP model was supported by our industrial partners Siemens Power and Gas Division, and Kraftanlagen Heidelberg (KAH). Improved DEMO plant configuration with IHTS/ESS for DEMO HCPB BB (18 sectors design), including conceptual designs of PHTS, IHTS/ESS and PCS is presented in this paper. The paper also presents detailed specifications of key components of the PCS, including sizing and preliminary cost estimates. So far the industrial components for IHTS/ESS and PHTS are still to be specified, so the foreseen IHTS/ESS and PHTS component designs are based primarily on design calculations performed by the project partners. It is worth to mention that no show stoppers were identified from the industry for the current BOP design.

Realization of the real equipment, availability and costs evaluation are foreseen in the next two years. The final design of DEMO BoP might still be different, depending on the further research and development within the Eurofusion Project.

\section{Topic}

A. Experimental Fusion Devices and Supporting Facilities

\section{Preferred presentation}

Oral

\section{Keywords}

DEMO, Balance of Plant, Energy Storage System, Helium Cooled Pebble Bed Breeding Blanket

I grant permission to publish on behalf of all authors of the contribution. 